\title{
SOME RESULTS ON ABSOLUTE CONTINUITY FOR UNBOUNDED JACOBI MATRICES
}

\author{
JOANNE DOMBROWSKI
}

Abstract. This brief paper presents several results which offer a useful approach for studying the absolute continuity of spectral measures associated with some Jacobi matrix operators. The operators are modeled as multiplication operators on a dense domain of a function space with a polynomial basis. Properties of these polynomials and commutator equations are used to obtain results on absolute continuity.

Mathematics subject classification (2010): 47B36, 47B15, 47B39.

Keywords and phrases: Jacobi matrices, absolute continuity, commutator equations.

\section{REFERENCES}

[1] J. BEREZANS KII, Expansions in Eigenfunctions of Self-adjoint Operators, translations of Mathematics Monographs, vol. 17, Amer. Math. Soc. Providence, R. I. 1968.

[2] J. DomBRows KI, A commutator approoach to absolute continuity for unbounded Jacobi operators, J. Math. Anal. Appl. 378 (2011), no. 1, 133-139.

[3] J. Dombrows KI, Jacobi matrices: eigebenvalues and spectral gaps, Methods of Spectral Analysis in Mathematical Physics, Operator Theory: Advances and Applications, vol. 186, 103-113, Birkhauser, Basil, 2008.

[4] J. Dombrows ki And S. Pedersen, Absolute continuity for unbounded Jacobi matrices with constant row sums, J. Math. Anal. Appl. 277 (2002), 695-713.

[5] S. Pedersen, Absolutely continuous Jacobi Operators, Proc. Amer. Math. Soc. 130 (2002), 23692376.

[6] C. R. Putnam, Commutation Properties of Hilbert Space Operators and Related Topics, Ergebnisse der Math., vol. 36, Springer-Verlag, Berlin, 1967. 